SLAC-PUB-15633

\title{
Klystron Modulator System Incorporating a Pulsed Depressed Collector*
}

\author{
Mark A. Kemp, Aaron Jensen, and Jeff Neilson \\ SLAC National Accelerator Laboratory, Menlo Park, CA USA
}

Applications utilizing high-power RF sources are increasingly placing a premium on electrical efficiency. In the case of particle accelerator laboratories, a large portion of the overall site electrical power is consumed by the RF systems. Therefore, new concepts for improving the efficiency of RF systems are desired.

SLAC National Accelerator Laboratory has undertaken a multi-faceted approach for reducing laboratory energy demand. As part of this effort, a pulsed depressed collector is under development to significantly improve the efficiency of RF systems. This device recovers energy from the klystron collector and feeds it back to the modulator for use on subsequent pulses. While depressed collectors have been utilized extensively in CW TWTs, gyrotrons, and some klystrons, use in pulsed systems is not widespread. For pulsed systems, a significant amount of energy can be wasted in the rise and fall times, which places a constraint upon the modulators.

The proposed pulsed depressed collector uses a novel feed-forward, charge-mode energy recovery scheme. An advantage of this is that no external power supplies are necessary for biasing of collector electrodes. In addition, substantial modification of the modulator is not required; energy can be simply recovered to almost any useful point in the system. Also, the only fundamental modification of the klystron needed is the replacement of the existing collector with a modified collector. Results indicate that for one particular RF system, the efficiency can be improved from

$37 \%$ to over $68 \%$. Finally, use of this collector in new RF system designs may reduce the constraints on modulator rise and fall time, potentially simplifying and reducing the cost of such systems.

This presentation will highlight aspects of a pulsed depressed collector design ${ }^{1,2}$ and focus on the power conversion chain from the collector back to the modulator. In particular, PIC simulations of the collector, SPICE models of the transformer and power converter, system layout diagrams, as well as several case studies will be presented.

${ }^{1}$ M. Kemp, A. Jensen, J. Neilson, "Pulsed Depressed Collector for High-Efficiency RF systems,” IVEC 2013.

${ }^{2}$ Patent pending. 